\title{
Anemia post-trapianto renale
}

\author{
A. Rosati \\ U.O. Nefrologia e Dialisi, Ospedale Campo di Marte, Lucca
}

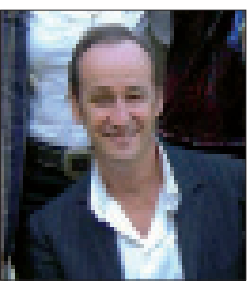

Il riscontro di anemia in corso di $\mathrm{CKD}$ è un evento assai frequente e conosciuto. E dovuta principalmente al deficit di eritropoietina (EPO), ma anche la EPO-resistenza, il deficit assoluto o relativo di ferro possono giocare un ruolo. L'anemia contribuisce in maniera rilevante alla sintomatologia che accompagna la malattia renale cronica ed effetti ben noti sono la peggiore qualità della vita, la ridotta resistenza alla fatica, il più elevato tasso di ospedalizzazione e la ridotta sopravvivenza dei pazienti in dialisi. Nonostante da circa vent'anni siano disponibili agenti stimolanti l'eritropoiesi (ESA) e siano ampiamente conosciute e condivise linee guida per il trattamento dell'anemia, la percentuale di pazienti che non raggiungono i livelli minimi attesi di $\mathrm{Hb}$ è inaspettatamente elevata. Ciò è dovuto all'inadeguatezza del trattamento da una parte e dalla resistenza al trattamento con ESA dall'altra.

Prevalenza e caratteristiche dell'anemia nel paziente trapiantato renale sono molto meno conosciute e molto scarsa è la letteratura sull'argomento al contrario di quanto si può affermare relativamente a CKD e dialisi. Solo negli ultimi anni si osserva un certo interesse intorno all'argomento, in particolare dopo che ci si è resi conto che la principale causa di perdita dell'organo trapiantato è la morte con rene funzionante e che tale mortalità è in gran parte dovuta a cause cardiovascolari. Del resto il rapporto fra anemia e mortalità cardiovascolare è stato ampiamente dimostrato per pazienti affetti da $\mathrm{CKD}$ e questo ha fatto prendere coscienza del ruolo che potrebbe svolgere anche nel paziente trapiantato.

Lapproccio al paziente trapiantato come paziente affetto da malattia renale cronica per lo più in stadio III non è tuttora sufficientemente condiviso vuoi perché solo una parte dei trapiantati renali riceve un follow-up nefrologico vuoi perché l'attenzione in questi pazienti è maggiormente focalizzata alla prevenzione e al trattamento dei fenomeni immunologici. Inoltre i dati accumulati nel paziente con $\mathrm{CKD}$ non sono automaticamente trasferi- bili nel paziente trapiantato. Vi sono alcuni aspetti che rendono il paziente trapiantato diverso dal paziente con $\mathrm{CKD}$ con pari funzione quali, ad esempio, la terapia immunosoppressiva, l'alterato stato infiammatorio e il periodo trascorso in trattamento dialitico.

\section{Epidemiologia}

La prevalenza di anemia post-trapianto riportata nelle varie casistiche è estremamente variabile in particolare perché molto eterogenea è la definizione che di volta in volta viene utilizzata. Altrettanto mutevole è la distanza dal trapianto in cui viene effettuata la rilevazione. Lo studio più ampio in questo ambito è il Transplant European Study on Anemia Management (TRESAM) Study (1). Questa cross-sectional survey che ha coinvolto 72 Centri Trapianto in 16 Paesi, definendo come anemia una $\mathrm{Hb}<13 \mathrm{~g} / \mathrm{dL}$ per i maschi e una $\mathrm{Hb}<12 \mathrm{~g} / \mathrm{dL}$ per le femmine, ha rilevato una prevalenza di anemia posttrapianto del $38,6 \%$ mentre la percentuale di pazienti in trattamento con ESA era del $17,8 \%$. In altre casistiche sono state riportate prevalenze più alte $o$ più basse in relazione ai succitati diversi criteri di valutazione ma in sintesi possiamo affermare che in una coorte composta da pazienti con varie anzianità di trapianto mediamente $1 / 3$ dei pazienti è anemico e circa $2 / 3$ dei pazienti trapiantati sviluppano anemia nel corso della vita del trapianto.

\section{Storia naturale}

La maggior parte dei pazienti in trattamento dialitico è oggi in trattamento con ESA e quindi oggi molto più che in passato, i pazienti arrivano al trapianto con livelli di $\mathrm{Hb}$ adeguati. Inoltre, nel periodo pre-trapianto, questi pazienti hanno ricevuto un numero di trasfusioni molto minore che in passato e questo, grazie alla minor frequenza di sensibilizzazioni, ha determinato un miglio- 
ramento della sopravvivenza a 5 anni del graft. È interessante notare anche che i pazienti iporesponsivi agli ESA hanno una ridotta sopravvivenza del trapianto (2). Il diffondersi del trapianto preemptive ha inoltre dimostrato che i pazienti che vengono sottoposti a trapianto prima dell'inizio della dialisi hanno meno probabilità di sviluppare anemia nel post-trapianto, forse in relazione alla miglior funzione renale che in genere contraddistingue questa categoria di pazienti.

Nell'immediato periodo post-trapianto si osserva un brusco calo dei livelli di $\mathrm{Hb}$ legato alle perdite connesse alle procedure chirurgiche e alle ripetute venopunture, al ritardo nella ripresa della funzione renale e ai fenomeni infiammatori legati a infezioni e rigetti. Questo calo raggiunge il minimo dopo 4 settimane circa dopodiché si ha un lento e progressivo recupero che si completa dopo circa 6 mesi-1 anno. Intorno al primo anno la maggior parte dei problemi del primo periodo sono scomparsi e si osserva la più bassa prevalenza di pazienti anemici della storia naturale del trapianto (3). A partire dal secondo anno correlato al ridursi del filtrato glomerulare si ha un progressivo e crescente incremento della prevalenza di anemia (4). Si è osservato che i pazienti che rientrano in dialisi dopo il fallimento di un trapianto sono anemici in una percentuale paragonabile a quella dei pazienti con CKD che non hanno mai ricevuto un follow-up nefrologico (late referral).

\section{Cause e fattori di rischio}

Nel primo periodo post-trapianto un raro, ma a volte prolungato, episodio di anemia si verifica come conseguenza di una reazione emolitica anti-Rh in riceventi di organi $\mathrm{Rh}$ positivi da donatori di sesso femminile Rrh negativi che hanno concepito un feto Rh positivo ed hanno sviluppato una risposta immunitaria anti-Rh. Queste donatrici a volte possono trasferire plasmacellule all'interno del trapianto, provocando una risposta immunitaria anti-Rh e successiva anemia emolitica nel ricevente (5). Il quadro è in genere autolimitante ed è trattato con trasfusioni e riduzione della terapia immunosoppressiva. Anche l'infezione da Parvovirus B19 è raramente causa di intensa anemia che insorge precocemente post-trapianto e che risente positivamente del trattamento con Ig per via endovenosa (6). Naturalmente la causa più frequente di anemia nel post-trapianto è il progressivo deteriorarsi della funzione dell'organo trapiantato con il venir meno della produzione endogena di EPO. Esiste una stretta associazione fra il livello di funzione renale e la prevalenza di anemia nei pazienti trapiantati così come nei pazienti con $\mathrm{CKD}$ ma a parità di funzione renale la pre- valenza è circa 10 volte superiore nei portatori di un trapianto renale. La riduzione del filtrato spiega quindi solo in parte la prevalenza di anemia e non esiste una stretta correlazione fra livelli di EPO e VGF (7). Molti altri fattori esercitano quindi un ruolo determinante, in particolare farmaci immunosoppressori, ACEI, ARB, lo stato infiammatorio cronico ecc. I farmaci immunosoppressori possono contribuire allo sviluppo di anemia con una serie di meccanismi: Azatioprina e MMF, agenti antiproliferativi, possono determinare una diretta inibizione del midollo, Ciclosporina e Tacrolimus vengono più spesso associati allo sviluppo di leucopenia ma talora sono stati associati anche allo sviluppo di anemia $(8,9)$, la terapia con Sirolimus è gravata da una prevalenza di anemia abbastanza elevata, soprattutto se associata a MMF (10), dose dipendente, probabilmente dovuta a una sorta di resistenza all'EPO nelle popolazioni eritroidi. Tradizionalmente ACEI e ARB hanno un effetto deprimente sui livelli di $\mathrm{Hb}$ anche nel paziente con $\mathrm{CKD}$, ma il paziente trapiantato ha una suscettibilità decisamente maggiore, forse per un più alto livello di attivazione del RAAS.

\section{Conseguenze}

Nel paziente con CKD l'associazione fra livelli di $\mathrm{Hb}$, mortalità e morbilità è ampiamente dimostrata ma per i motivi succitati non è automaticamente trasferibile nel paziente trapiantato. Molti studi hanno provato ad indagare il rapporto fra $\mathrm{Hb}$ e mortalità cardiovascolare e generale e fra $\mathrm{Hb}$ e sopravvivenza del graft. Si tratta per lo più di studi retrospettivi o osservazionali con varie limitazioni e livelli di evidenza bassi (11). I risultati che ne emergono sono contraddittori in termini sia di mortalità sia di sopravvivenza del graft. Non si può comunque ignorare che quella CV è di gran lunga la prima causa di morte ed è altamente probabile che almeno una parte di questa sia riconducibile alle conseguenze dell'anemia (12). Il legame con la sopravvivenza dell'organo è certamente più incerto e necessita di ulteriori conferme.

\section{Trattamento}

Non è ad oggi dimostrato che gli studi condotti su pazienti con CKD siano automaticamente trasferibili al paziente trapiantato renale. Vi sono comunque alcuni studi che hanno dimostrato l'efficacia del trattamento con ESAs nella correzione dell'anemia nel paziente trapiantato renale (5). Se teniamo conto del fatto che i livelli di EPO sono più elevati della norma nei primi 3 mesi dal trapianto, pare ovvio iniziare il trattamento a partire dal 
terzo mese. Non vi sono informazioni circa i target di $\mathrm{Hb}$ da mantenere nel paziente trapiantato. È verosimile che, come accade nel paziente con $\mathrm{CKD}$, il cercare di raggiungere livelli di $\mathrm{Hb}>12,5-13 \mathrm{~g} / \mathrm{dL}$ a prezzo di elevati dosaggi di ESAs sia controproducente e potenzialmente pericoloso. La manipolazione della terapia immunosoppressiva finalizzata alla gestione dell'anemia richiede molta cautela non solo per i rischi legati alla funzionalità del graft ma anche perché non è mai facile quantificare il peso della terapia immunosoppressiva sui livelli di $\mathrm{Hb}$ e capire quale singolo farmaco sia maggiormente responsabile. La sospensione dei farmaci bloccanti il RAAS è invece un intervento talora efficace di fronte a un quadro di anemia severa e non responsiva agli ESAs. Non vi sono indicazioni circa la maneggevolezza e l'efficacia dei singoli ESAs per il trattamento dell'anemia nel trapiantato renale ma di fatto tutti gli agenti disponibili possono essere utilizzati. A oggi comunque il problema principale è la scarsa attenzione che viene riservata al problema, visto che meno della metà dei pazienti eligibili per un trattamento riceve in realtà un ESA.

\section{Conclusioni}

L'anemia post-trapianto è oggi una problematica clinica largamente sottostimata e non adeguatamente trattata. I target terapeutici riconosciuti per la CKD sono nel trapianto più difficilmente raggiungibili perché l'anemia riconosce una genesi multifattoriale. Il trattamento con ESAs è meno efficace e i tempi di correzione più lunghi. Quasi tutti i farmaci immunosoppressori possono influenzare i livelli di $\mathrm{Hb}$. Per tutti questi motivi il trattamento dovrebbe essere fatto da un nefrologo particolarmente esperto in questo settore mentre invece molto spesso sono altre figure specialistiche che gestiscono il follow-up ambulatoriale di questi pazienti. Il potenziale effetto sulla mortalità cardiovascolare e il rischio di influenzare pesantemente le condizioni cliniche al momento del rientro in dialisi dovrebbero essere di per sé validi motivi per presidiare in maniera più adeguata questa delicata complicanza del trapianto renale.

\section{Bibliografia}

1. Vanrenterghem Y, Ponticelli C, Morales JM, et al. Prevalence and management of anemia in renal transplant recipients: A European survey. Am J Transplant 2003; 3: 835-45.

2. Campise M, Mikhail A, Quaschning T, et al. Impact of pre-transplant anaemia correction and erythropoietin resistance on long-term graft survival. Nephrol Dial Transplant 2005; 20 (Suppl 8): viii8-viii12.

3. Mix TC, Kazmi W, Khan S, et al. Anemia: A continuing problem following kidney transplantation. Am J Transplant 2003; 3: 1426-33.

4. Afzali B, Al-Khoury S, Shah N, et al. Anemia after renal transplantation. Am J Kidney Dis 2006; 48: 519-36.

5. Winkelmayer WC, Chandraker A. Posttransplantation Anemia: Management and Rationale. Clin J Am Soc Nephrol 2008; 3(Suppl): S49-S55.

6. Egbuna O, Zand MS, Arbini A, et al. A cluster of parvovirus B19 infections in renal transplant recipients: A prospective case series and review of the literature. Am J Transplant 2006; 6: 225-31.

7. Sun $\mathrm{CH}$, Ward HJ, Paul WL, et al. Serum erythropoietin levels after renal transplantation. N Engl J Med 1989; 321: 151-7.

8. Winkelmayer WC, Kewalramani R, Rutstein M, et al Pharmacoepidemiology of anemia in kidney transplant recipients. J Am Soc Nephrol 2004; 15: 1347-52.

9. Chadban SJ, Baines L, Polkinghorne K, et al. Anemia after kidney transplantation is not completely explained by reduced kidney function. Am J Kidney Dis 2007; 49: 301-9.

10. Augustine JJ, Knauss TC, Schulak JA, et al. Comparative effects of sirolimus and mycophenolate mofetil on erythropoiesis in kidney transplant patients. Am J Transplant 2004; 4: 2001-6.

11. Molnar MZ, Czira M, Ambrus C, et al. Anemia is associated with mortality in kidney-transplanted patients patients: A prospective cohort study. Am J Transplant 2007; 7: 818-24.

12. Rigatto C, Parfrey P, Foley R, et al. Congestive heart failure in renal transplant recipients: Risk factors, outcomes, and relationship with ischemic heart disease. J Am Soc Nephrol 2002; 13: 1084-90.

\section{Indirizzo degli Autori:}

Alberto Rosati, MD

U.O. Nefrologia e Dialisi

Ospedale Campo di Marte

Via dell'Ospedale 1

55100 Lucca

a.rosati@usl2.toscana.it 